Portland State University

PDXScholar

Electrical and Computer Engineering Faculty

Publications and Presentations

Electrical and Computer Engineering

$1-1-1993$

\title{
Pump and signal cross saturation in cw dye lasers
}

Lee W. Casperson

Portland State University

A. C. Wilson

W. J. Sandle

Follow this and additional works at: https://pdxscholar.library.pdx.edu/ece_fac

Part of the Electrical and Computer Engineering Commons

Let us know how access to this document benefits you.

\section{Citation Details}

Casperson, L. W., Wilson, A. C., \& Sandle, W. J. (1993). Pump and signal cross saturation in cw dye lasers. Journal Of Applied Physics, 73(3), 1049.

This Article is brought to you for free and open access. It has been accepted for inclusion in Electrical and Computer Engineering Faculty Publications and Presentations by an authorized administrator of PDXScholar. Please contact us if we can make this document more accessible: pdxscholar@pdx.edu. 


\title{
Pump and signal cross saturation in cw dye lasers
}

\author{
Lee W. Casperson, ${ }^{\text {a) }}$ A. C. Wilson, and W. J. Sandle \\ Department of Physics, University of Otago, P.O. Box 56, Dunedin, New Zealand
}

(Received 6 July 1992; accepted for publication 6 October 1992)

It is well known that the signal output intensity from a dye laser oscillator depends directly on the pump intensity. The opposite effect, the dependence of the transmitted pump intensity on the signal intensity, is studied in detail. The theoretical results are compared with experimental data obtained using an argon-laser-pumped rhodamine $6 \mathrm{G}$ dye laser.

\section{INTRODUCTION}

Dye lasers are often operated using other lasers as their pump sources. Typically, both the pump and signal fields are linearly polarized, and a rigorous study of the performance of these lasers requires an understanding of the interaction of such fields with the orientationally distributed dye molecules. There have been several studies of the polarization characteristics of dye laser amplifiers and oscillators, ${ }^{1-10}$ and recently the dependence of the dye laser oscillator output power on the pump power and polarization direction has been studied in detail. ${ }^{11}$ As one would anticipate, the highest laser efficiency results when the pump and signal polarization directions are parallel. Because of their wide frequency tuning range and potentially high-frequency stability, $\mathrm{cw}$ dye lasers have found many practical applications. Any developments that might improve the understanding and performance of these somewhat complicated systems would have substantial practical consequences.

In an idealized four-level laser system, the majority of the lasing atoms or molecules are understood to always be in the ground state. Thus, the pump rate for the laser transition is almost independent of any spontaneous or stimulated transitions that might occur. This simplifying assumption underlies almost all treatments of dye lasers, but it seems never to have been subjected to detailed theoretical analysis or experimental verification. The purpose of this article is to look in detail at the effects of the signal transition on the populations and transition rates associated with the pump transition. It is shown theoretically that saturation of the signal transition in a dye laser might lead to a significant modification of the populations associated with the pump transition. Experimentally, it is found that the strength of the pump power that is transmitted through the dye in a dye jet laser oscillator is reduced significantly when the dye is saturated by the oscillator signal.

A basic theoretical model for saturation in dye lasers is developed in Sec. II. This model differs from most previous studies in that the possible effects of the lasing signal on the absorption of the pump transition are fully included. It is found that, to the order of approximation employed here,

\footnotetext{
'Permanent address: Department of Electrical Engineering, Portland State University, P. O. Box 751, Portland, OR 97207-0751.
}

saturation by the signal in a dye laser oscillator leads to a transmitted pump power that is proportional to the incident pump power. The theoretical results are compared with experimental data obtained with an argon-laserpumped rhodamine 6G dye laser in Sec. III, and good agreement is obtained.

\section{THEORY}

The basic semiclassical model on which this study is based was described in detail in Ref. 8. The advantage of a semiclassical model for this purpose is that the vector properties of the pump and signal fields are automatically included, and it is not necessary to postulate a polarization dependence of the absorption and emission cross sections. In a rate equation model the fields are usually represented more simply as intensities or photon densities. The energylevel model used in this analysis is the four-level system shown in Fig. 1. Pump absorption takes place between levels zero and three, while stimulated emission takes place between levels two and one. The coupling between the absorption levels and the emission levels is taken to be nonradiative relaxation with a relaxation time $\tau_{3}$ between levels three and two and $\tau_{1}$ between levels one and zero. Levels three and two are members of a single vibrational manifold as are levels zero and one.

The procedure for setting up the semiclassical model corresponding to Fig. 1 is well known, and a more detailed discussion is given in Ref. 8. Our interest here is in the cw operation of the laser amplifier, and in this case the population differences $D_{p}=\rho_{00}-\rho_{33}$ of the pump transition and $D_{s}=\rho_{22}-\rho_{11}$ of the signal transition can be expressed explicitly in terms of the fields. For one class of dipoles the population differences are ${ }^{8}$

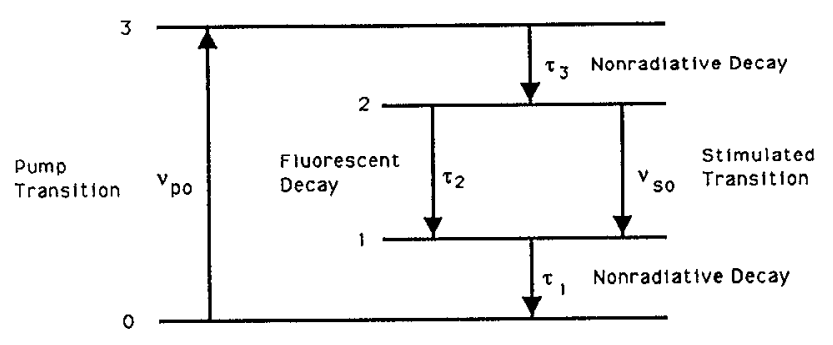

FIG. 1. Energy-level model used in the dye laser analysis. 


$$
\begin{aligned}
& D_{p}(\theta, \phi, z)=\frac{1+\tau_{2} \beta_{s}(\theta, \phi, z)}{1+\left(2 \tau_{3}+\tau_{2}+\tau_{1}\right) \beta_{p}(\theta, \phi, z)+\tau_{2} \beta_{s}(\theta, \phi, z)+2 \tau_{2}\left(\tau_{3}+\tau_{1}\right) \beta_{p}(\theta, \phi, z) \beta_{s}(\theta, \phi, z)} \\
& D_{s}(\theta, \phi, z)=\frac{\left(\tau_{2}-\tau_{1}\right) \beta_{p}(\theta, \phi, z)}{1+\left(2 \tau_{3}+\tau_{2}+\tau_{1}\right) \beta_{p}(\theta, \phi, z)+\tau_{2} \beta_{s}(\theta, \phi, z)+2 \tau_{2}\left(\tau_{3}+\tau_{1}\right) \beta_{p}(\theta, \phi, z) \beta_{s}(\theta, \phi, z)}
\end{aligned}
$$

The angle variables $\theta$ and $\phi$ indicate the orientation of the particular pump or signal dipole class with respect to the $x$ axis. The field variables are

$$
\begin{aligned}
& \beta_{p}(\theta, \phi, z)=\left|\mu_{p}\right|^{2}\left|\mathbf{E}_{p}^{\prime}(z) \cdot \hat{e}_{p}\right|^{2} L\left(v_{p}\right) / 4 \hbar^{2}, \\
& \beta_{s}(\theta, \phi, z)=\left|\mu_{s}\right|^{2}\left|\mathbf{E}_{s}^{\prime}(z) \cdot \hat{e}_{s}\right|^{2} L\left(v_{s}\right) / 4 \hbar^{2},
\end{aligned}
$$

where $\left|\mu_{p}\right|$ and $\left|\mu_{s}\right|$ are the magnitudes of the pump and signal dipole moments, $\mathbf{E}_{p}^{\prime}(z)$ and $\mathbf{E}_{s}^{\prime}(z)$ are the complex vector amplitudes of the pump and signal electric fields, $\hat{e}_{p}$ and $\hat{e}_{s}$ are unit vectors in the directions of the pump and signal dipole moments, and $L\left(v_{p}\right)$ and $L\left(v_{s}\right)$ are the normalized Lorentzian line-shape functions

$$
\begin{aligned}
& L\left(v_{p}\right)=\frac{2 / \pi \Delta v_{p}}{1+\left[2\left(v_{p}-v_{p 0}\right) / \Delta v_{p}\right]^{2}}, \\
& L\left(v_{s}\right)=\frac{2 / \pi \Delta v_{s}}{1+\left[2\left(v_{s}-v_{s 0}\right) / \Delta v_{s}\right]^{2}} .
\end{aligned}
$$

In these functions $v_{p}$ and $v_{s}$ represent the actual frequencies of the pump and signal fields, $v_{p 0}$ and $v_{s 0}$ are the center frequencies of the pump and signal transitions, and $\Delta v_{p}$ and $\Delta v_{s}$ are the full widths at half-maximum of these transitions. These widths are related to the coherence times of the transitions by $\Delta v_{p}=\left(\pi T_{p}\right)^{-1}$ and $\Delta v_{s}=\left(\pi T_{s}\right)^{-1}$.

The real electric fields are assumed to propagate harmonically in the $z$ direction and are thus related to the complex field amplitudes in Eqs. (3) and (4) by the equations

$$
\begin{aligned}
& \mathbf{E}_{p}(z, t)=\frac{1}{2} \mathbf{E}_{p}^{\prime}(z) \exp \left[i\left(k_{p} z-\omega_{p} t\right)\right]+\text { c.c. } \\
& \mathbf{E}_{s}(z, t)=\frac{1}{2} \mathbf{E}_{s}^{\prime}(z) \exp \left[i\left(k_{s} z-\omega_{s} t\right)\right]+\text { c.c. }
\end{aligned}
$$

where $k_{p}$ and $k_{s}$ are the propagation constants and $\omega_{p}$ and $\omega_{s}$ the radian frequencies of the pump and signal fields. The notation c.c. refers to the complex conjugate of the preceding terms. The essence of the cross-saturation behavior is evident already in Eqs. (1) and (2), where the population differences are seen to depend on both the pump and signal fields. In the following analysis these population differences are combined with Maxwell's equations to obtain more explicit expressions for the relationships between the pump and signal fields in a saturating laser amplifier.

The differential equations that $\mathbf{E}_{p}^{\prime}(z)$ and $\mathbf{E}_{s}^{\prime}(z)$ satisfy are derived from the vector wave equation, and the results can be written

$$
\frac{d \mathbf{E}_{p}^{\prime}(z)}{d z}+\frac{\gamma_{p}}{2} \mathbf{E}_{p}^{\prime}(z)=i\left(\frac{\mu}{\epsilon}\right)^{1 / 2} \omega_{p} \mathbf{P}_{p}^{\prime}(z),
$$

$$
\frac{d \mathbf{E}_{s}^{\prime}(z)}{d z}+\frac{\gamma_{s}}{2} \mathbf{E}_{s}^{\prime}(z)=i\left(\frac{\mu}{\epsilon}\right)^{1 / 2} \omega_{s} \mathbf{P}_{s}^{\prime}(z),
$$

where the higher-order space derivatives of the slowly varying amplitudes have been neglected, and the rotatingwave approximation has been employed. Thus the population differences are functions of space through the fielddependent $\beta$ parameters in Eqs. (1) and (2). The propagation constants in Eqs. (7) and (8) have been related to the frequencies by $k_{p}=(\mu \epsilon)^{1 / 2} \omega_{p}$ and $k_{s}$ $=(\mu \epsilon)^{1 / 2} \omega_{s}$, and $\gamma_{p}$ and $\gamma_{s}$ represent all distributed losses incurred by the pump and signal fields, respectively. The complex polarization amplitudes are defined by the equations

$$
\begin{aligned}
& \mathbf{P}_{p}(z, t)=\mathbf{P}_{p}^{\prime}(z) \exp \left[i\left(k_{p} z-\omega_{p} t\right)\right]+\text { c.c., } \\
& \mathbf{P}_{s}(z, t)=\mathbf{P}_{s}^{\prime}(z) \exp \left[i\left(k_{s} z-\omega_{s} t\right)\right]+\text { c.c. },
\end{aligned}
$$

and these amplitudes are related to the field and molecular variables by

$$
\begin{aligned}
\mathbf{P}_{p}^{\prime}(z)= & -\frac{\left|\mu_{p}\right|^{2} L\left(v_{p}\right)\left[\left(\omega_{p}-\omega_{p 0}\right) T_{p}-i\right]}{4 \hbar} \\
& \times \int_{0}^{2 \pi} \int_{0}^{\pi} n_{p}(\theta, \phi) D_{p}(\theta, \phi, z)\left(\mathbf{E}_{p}^{\prime} \cdot \hat{e}_{p}\right) \hat{e}_{p} \\
& \times \sin \theta d \theta d \phi, \\
\mathbf{P}_{s}^{\prime}(z)= & \frac{\left|\mu_{s}\right|^{2} L\left(v_{s}\right)\left[\left(\omega_{s}-\omega_{s 0}\right) T_{s}-i\right]}{4 \hbar} \\
& \times \int_{0}^{2 \pi} \int_{0}^{\pi} n_{s}(\theta, \phi) D_{s}(\theta, \phi, z)\left(\mathbf{E}_{s}^{\prime} \cdot \hat{e}_{s}\right) \hat{e}_{s} \\
& \times \sin \theta d \theta d \phi,
\end{aligned}
$$

where $n_{p}(\theta, \phi)$ and $n_{s}(\theta, \phi)$ are the orientational distributions of the pump and signal dipoles.

The model that has just been described provides a basis for a variety of studies concerning $\mathrm{cw}$ amplification and oscillation in systems with orientationally distributed active molecules, and several solutions were explored in Refs. 8 and 9 and elsewhere. Our purpose here is to emphasize cross-saturation effects, and so we focus on the most common and one of the simplest field and molecule configurations. In particular, it is usual and most efficient to have the pump and signal polarizations parallel to each other, ${ }^{11}$ and we will definc this common direction to be the $x$ direction. The unit vectors $\hat{e}_{p}$ and $\hat{e}_{s}$ can be expressed in terms of the coordinate unit vectors $\hat{e}_{x}, \hat{e}_{y}$ and $\hat{e}_{z}$ by means of the relationships 


$$
\begin{aligned}
& \hat{e}_{p}=\left(\hat{e}_{p} \cdot \hat{e}_{x}\right) \hat{e}_{x}+\left(\hat{e}_{p} \cdot \hat{e}_{y}\right) \hat{e}_{y}+\left(\hat{e}_{p} \cdot \hat{e}_{z}\right) \hat{e}_{z} \\
& \hat{e}_{s}=\left(\hat{e}_{s} \cdot \hat{e}_{x}\right) \hat{e}_{x}+\left(\hat{e}_{s} \cdot \hat{e}_{y}\right) \hat{e}_{y}+\left(\hat{e}_{s} \cdot \hat{e}_{z}\right) \hat{e}_{z}
\end{aligned}
$$

When these formulas are used with Eqs. (13) and (14), the $x$ components of the polarization are given by

$$
\begin{aligned}
P_{p x}^{\prime}(z)= & -\frac{\left|\mu_{p}\right|^{2} L\left(v_{p}\right)\left[\left(\omega_{p}-\omega_{p 0}\right) T_{p}-i\right]}{4 \hbar} E_{p x}^{\prime}(z) \\
& \times \int_{0}^{2 \pi} \int_{0}^{\pi} n_{p}(\theta, \phi) D_{p}(\theta, \phi, z) \cos ^{2} \theta \sin \theta d \theta d \phi, \\
P_{s x}^{\prime}(z)= & \frac{\left|\mu_{s}\right|^{2} L\left(v_{s}\right)\left[\left(\omega_{s}-\omega_{s 0}\right) T_{s}-i\right]}{4 \hbar} E_{s x}^{\prime}(z) \\
& \times \int_{0}^{2 \pi} \int_{0}^{\pi} n_{s}(\theta, \phi) D_{s}(\theta, \phi, z) \cos ^{2} \theta \sin \theta d \theta d \phi .
\end{aligned}
$$

Also, the $\beta$ parameters of Eqs. (3) and (4) that appear in the pump and signal population differences can now be written

$$
\begin{aligned}
& \beta_{p}(\theta, \phi, z)=\left|\mu_{p}\right|^{2}\left|E_{p x}^{\prime}(z)\right|^{2} \cos ^{2} \theta \quad L\left(v_{p}\right) / 4 \hbar^{2}, \\
& \beta_{s}(\theta, \phi, z)=\left|\mu_{s}\right|^{2}\left|E_{s x}^{\prime}(z)\right|^{2} \cos ^{2} \theta L\left(v_{s}\right) / 4 \hbar^{2} .
\end{aligned}
$$

With these values of $\beta_{p}$ and $\beta_{s}$, the population differences in Eqs. (1) and (2) are a bit simpler, and these simplified forms may be substituted into the polarizations in Eqs. (17) and (18). The reduced model is completed by substituting the polarizations into the wave equations given as Eqs. (9) and (10).

In most visible wavelength lasers the absorption and emission dipoles are parallel $\left(\hat{e}_{p}=\hat{e}_{s}\right)$ and thus have the same orientational distribution $\left(n_{p}=n_{s}=n\right)$. It is also assumed here that the pump and signal fields are close to the center frequencies of their respective transitions, and hence the Lorentzian functions given in Eqs. (5) and (6) reduce to

$$
\begin{aligned}
& L\left(v_{p}\right)=2 / \pi \Delta v_{p}, \\
& L\left(v_{s}\right)=2 / \pi \Delta v_{s}
\end{aligned}
$$

In this case it is possible to assume that the field amplitudes are real and the polarization amplitudes imaginary. Then the polarization amplitudes of Eqs. (17) and (18) may be substituted into the field equations given in Eqs. (9) and (10) to obtain

$$
\begin{aligned}
& \frac{d E_{p x}^{\prime}(z)}{d z}+\frac{\gamma_{p}}{2} E_{p x}^{\prime}(z)=-\frac{\alpha_{x}}{2} E_{p x}^{\prime}(z), \\
& \frac{d E_{s x}^{\prime}(z)}{d z}+\frac{\gamma_{s}}{2} E_{s x}^{\prime}(z)=\frac{g_{x}}{2} E_{s x}^{\prime}(z),
\end{aligned}
$$

where the absorption and gain coefficients are given by

$$
\begin{aligned}
\alpha_{x}= & \left(\frac{\mu}{\epsilon}\right)^{1 / 2} \frac{2 v_{p}\left|\mu_{p}\right|^{2}}{\hbar \Delta v_{p}} \\
& \times \int_{0}^{2 \pi} \int_{0}^{\pi} n(\theta, \phi) D_{p}(\theta, \phi, z) \cos ^{2} \theta \sin \theta d \theta d \phi,
\end{aligned}
$$

$$
\begin{aligned}
g_{x}= & \left(\frac{\mu}{\epsilon}\right)^{1 / 2} \frac{2 v_{s}\left|\mu_{s}\right|^{2}}{\hbar \Delta v_{s}} \\
& \times \int_{0}^{2 \pi} \int_{0}^{\pi} n(\theta, \phi) D_{s}(\theta, \phi, z) \cos ^{2} \theta \sin \theta d \theta d \phi .
\end{aligned}
$$

To be specific, it is also assumed that the relaxation times for the nonradiative decays from levels three and one are much less than the effective lifetime of the upper laser state, level two. In a dye, the vibrational relaxation times are on the order of picoseconds, while the lifetime of the emitting level ranges from tens of picoseconds to several nanoseconds, depending on the magnitude of the stimulating field. Therefore, the population differences given in Eqs. (1) and (2) reduce to

$$
\begin{aligned}
& D_{p}(\theta, \phi, z)=\frac{1+\Phi_{s}^{2}(z) \cos ^{2} \theta}{1+\left[\Phi_{p}^{2}(z)+\Phi_{s}^{2}(z)\right] \cos ^{2} \theta} \\
& D_{s}(\theta, \phi, z)=\frac{\Phi_{p}^{2}(z) \cos ^{2} \theta}{1+\left[\Phi_{p}^{2}(z)+\Phi_{s}^{2}(z)\right] \cos ^{2} \theta}
\end{aligned}
$$

where the dimensionless field amplitudes are given by

$$
\begin{aligned}
& \Phi_{p}(z)=\left(\frac{\tau_{2}}{2 \pi \Delta v_{p}}\right)^{1 / 2} \frac{\left|\mu_{p}\right| E_{p x}^{\prime}}{\hbar}, \\
& \Phi_{s}(z)=\left(\frac{\tau_{2}}{2 \pi \Delta v_{s}}\right)^{1 / 2} \frac{\left|\mu_{s}\right| E_{s x}^{\prime}}{\hbar}
\end{aligned}
$$

These dimensionless field amplitudes may also be related to the intensities of the pump and signal fields by

$$
\begin{aligned}
& \Phi_{p}^{2}(z)=\tau_{2} \sigma_{p} I_{p}(z) / h v_{p}, \\
& \Phi_{s}^{2}(z)=\tau_{2} \sigma_{s} I_{s}(z) / h v_{s}
\end{aligned}
$$

where $\sigma_{p}$ and $\sigma_{s}$ are the absorption and emission cross sections given by

$$
\begin{gathered}
\sigma_{p}=\frac{2 v_{p} n_{0}\left|\mu_{p}\right|^{2}}{\epsilon c \hbar \Delta v_{p}}, \\
\sigma_{s}=\frac{2 v_{s} n_{0}\left|\mu_{s}\right|^{2}}{\epsilon c \hbar \Delta v_{s}} .
\end{gathered}
$$

In rhodamine $6 \mathrm{G}$ the absorption and stimulated emission cross sections are approximately equal. ${ }^{12}$ In terms of these cross sections the absorption and gain coefficients from Eqs. (25) and (26) are

$$
\alpha_{x}(z)=\sigma_{p} \int_{0}^{2 \pi} \int_{0}^{\pi} n(\theta, \phi) D_{p}(\theta, \phi, z) \cos ^{2} \theta \sin \theta d \theta d \phi,
$$


$g_{x}(z)=\sigma_{s} \int_{0}^{2 \pi} \int_{0}^{\pi} n(\theta, \phi) D_{s}(\theta, \phi, z) \cos ^{2} \theta \sin \theta d \theta d \phi$.

Our main interest here concerns dye laser media that have an isotropic distribution of dipole orientations. This situation would correspond to most ordinary dye lasers, where the active molecules are suspended with random orientations in liquid or solid media and the applied fields are too weak to cause any molecular alignment. For an isotropic molecular distribution the function $n(\theta, \phi)$ is simply

$$
n(\theta, \phi)=N / 4 \pi
$$

where $N$ is the total density of dye molecules. Since both the orientational distribution and the population differences in Eqs. (27) and (28) are now independent of the angle $\phi$, the absorption and gain coefficients from Eqs. (35) and (36) can be reduced to

$$
\begin{aligned}
& \alpha_{x}(z)=N \sigma_{p} \int_{0}^{1} \frac{1+\Phi_{s}^{2}(z) \chi^{2}}{1+\left[\Phi_{p}^{2}(z)+\Phi_{s}^{2}(z)\right] \chi^{2}} \chi^{2} d \chi, \\
& g_{x}(z)=N \sigma_{s} \int_{0}^{1} \frac{\Phi_{p}^{2}(z) \chi^{2}}{1+\left[\Phi_{p}^{2}(z)+\Phi_{s}^{2}(z)\right] \chi^{2}} \chi^{2} d \chi,
\end{aligned}
$$

where $\chi$ represents $\cos \theta$. These integrals can be performed and the results are

$$
\begin{aligned}
\alpha_{x}(z)= & N \sigma_{p}\left[\frac{1}{3}-\Phi_{p}^{2}(z)\left(\frac{1}{3\left[\Phi_{p}^{2}(z)+\Phi_{s}^{2}(z)\right]}\right.\right. \\
& -\frac{1}{\left[\Phi_{p}^{2}(z)+\Phi_{s}^{2}(z)\right]^{2}} \\
& \left.\left.+\frac{\tan ^{-1}\left[\Phi_{p}^{2}(z)+\Phi_{s}^{2}(z)\right]^{1 / 2}}{\left[\Phi_{p}^{2}(z)+\Phi_{s}^{2}(z)\right]^{5 / 2}}\right)\right], \\
g_{x}(z)= & N \sigma_{s} \Phi_{p}^{2}(z)\left(\frac{1}{3\left[\Phi_{p}^{2}(z)+\Phi_{s}^{2}(z)\right]}\right. \\
& -\frac{1}{\left[\Phi_{p}^{2}(z)+\Phi_{s}^{2}(z)\right]^{2}} \\
& \left.+\frac{\tan ^{-1}\left[\Phi_{p}^{2}(z)+\Phi_{s}^{2}(z)\right]^{1 / 2}}{\left[\Phi_{p}^{2}(z)+\Phi_{s}^{2}(z)\right]^{5 / 2}}\right) .
\end{aligned}
$$

These gain and loss expressions may be substituted into Eqs. (23) and (24), and the resulting differential equations are a complete set governing the pump and signal fields in a dye laser medium. Those equations may, in principle, be integrated subject to the input conditions of the pump and signal fields.

It follows from Eqs. (40) and (41) that the saturated absorption and gain coefficients in a dye laser amplifier are related to each other by the simple formula

$$
\frac{\alpha_{x}(z)}{N \sigma_{p}}=\frac{1}{3}-\frac{g_{x}(z)}{N \sigma_{s}} .
$$

If distributed losses are negligible $\left(\gamma_{p}=\gamma_{s}=0\right)$, this formula may be combined with Eqs. (23) and (24) to obtain

$$
-\frac{1}{N \sigma_{p} \Phi_{p}^{2}(z)} \frac{d \Phi_{p}^{2}(z)}{d z}=\frac{1}{3}-\frac{1}{N \sigma_{s} \Phi_{s}^{2}(z)} \frac{d \Phi_{s}^{2}(z)}{d z} .
$$

This equation can be integrated for an amplifier of length $z$, and the result can be written

$$
\frac{\Phi_{p}^{2}(z)}{\Phi_{p}^{2}(0)}=\left(\frac{\Phi_{s}^{2}(z)}{\Phi_{s}^{2}(0)}\right)^{\sigma_{p} / \sigma_{s}} \exp \left(-\alpha_{x 0} z\right),
$$

where from Eq. (38) the unsaturated pump absorption coefficient is given by

$$
\alpha_{x 0}=N \sigma_{p} \int_{0}^{1} \chi^{2} d \chi=\frac{N \sigma_{p}}{3} .
$$

Equation (44) could now be used to eliminate $\Phi_{p}(z)$ from Eq. (41) or $\Phi_{s}(z)$ from Eq. (40). Thus, the problem of a dye laser amplifier with cross saturation has been reduced to a matter of a single numerical integration. As shown in the Appendix, the corresponding equation for a unidirectional molecular distribution can be integrated analytically.

In terms of the intensities introduced in Eqs. (31) and (32), Eq. (44) can also be written

$$
\frac{I_{p}(z)}{I_{p}(0)}=\left(\frac{I_{s}(z)}{I_{s}(0)}\right)^{\sigma_{p} / \sigma_{s}} \exp \left(-\alpha_{x 0} z\right)
$$

This result provides an explicit formula for the transmitted pump intensity in terms of the unsaturated absorption and the other pump and signal intensities, and a comparison with experiment is given in the following section. A slight simplification of Eq. (46) occurs if the absorption and emission cross sections are considered to be equal.

It is also of interest to consider the propagation of the pump field in a dye laser medium when the signal field is set to zero. It follows from Eqs. (23) and (40) that the pump field in this case is governed by

$$
\begin{aligned}
-\frac{1 d \Phi_{p}^{2}(z)}{\Phi_{p}^{2}(z) d z}= & N \sigma_{p}\left[\frac{1}{3}-\Phi_{p}^{2}(z)\left(\frac{1}{3 \Phi_{p}^{2}(z)}\right.\right. \\
& \left.\left.-\frac{1}{\left[\Phi_{p}^{2}(z)\right]^{2}}+\frac{\tan ^{-1}\left[\Phi_{p}(z)\right]}{\left[\Phi_{p}^{2}(z)\right]^{5 / 2}}\right)\right] \\
= & N \sigma_{p}\left(\frac{1}{\Phi_{p}^{2}(z)}-\frac{\tan ^{-1}\left[\Phi_{p}(z)\right]}{\Phi_{p}^{3}(z)}\right) .
\end{aligned}
$$

In terms of the unsaturated loss, this equation can also be written

$$
\frac{d \Phi_{p}^{2}(z)}{d z}=-3 \alpha_{x 0}\left(1-\frac{\tan ^{-1}\left[\Phi_{p}(z)\right]}{\Phi_{p}(z)}\right) .
$$

This equation can be integrated numerically to obtain the $z$ dependence of the field amplitude in a saturating dye medium in which no laser signal field is present.

In most practical dye laser media there is only slight saturation of the pump absorption transition, and in this case Eq. (48) can be simplified. If $\Phi_{p}(z)$ is assumed to be 
small compared to unity, the inverse tangent function can be expanded in a power series; and the absorption equation becomes

$$
\begin{aligned}
\frac{d \Phi_{p}^{2}(z)}{d z}= & -3 \alpha_{x 0}\left[1-\frac{1}{\Phi_{p}(z)}\right. \\
& \left.\times\left(\Phi_{p}(z)-\frac{1}{3} \Phi_{p}^{3}(z)+\frac{1}{5} \Phi_{p}^{5}(z)-\cdots\right)\right] \\
\approx & -\alpha_{x 0} \Phi_{p}^{2}(z)\left(1-\frac{3}{5} \Phi_{p}^{2}(z)\right) \approx-\frac{\alpha_{x 0} \Phi_{p}^{2}(z)}{1+\frac{3}{5} \Phi_{p}^{2}(z)}
\end{aligned}
$$

This simplified equation can be integrated analytically, and the result is

$$
\log _{e}\left(\frac{\Phi_{p}^{2}(l)}{\Phi_{p}^{2}(0)}\right)+\frac{3}{5}\left[\Phi_{p}^{2}(l)-\Phi_{p}^{2}(0)\right]=-\alpha_{x 0} l
$$

Exponentiating both sides lead to

$$
\frac{\Phi_{p}^{2}(l)}{\Phi_{p}^{2}(0)} \exp \left(\frac{3}{5}\left[\Phi_{p}^{2}(l)-\Phi_{p}^{2}(0)\right]\right)=\exp \left(-\alpha_{x 0} l\right)
$$

Using again the assumption of little pump saturation, the exponential on the left-hand side of Eq. (50) can be expanded yielding

$$
\frac{\Phi_{p}^{2}(l)}{\Phi_{p}^{2}(0)}\left(1+\frac{3}{5}\left[\Phi_{p}^{2}(l)-\Phi_{p}^{2}(0)\right]\right)=\exp \left(-\alpha_{x 0} l\right)
$$

Equation (52) can be considered to be a quadratic equation for the output pump intensity, and the useful solution of this quadratic is

$$
\Phi_{p}^{2}(l)=\frac{-\left[1-\frac{3}{5} \Phi_{p}^{2}(0)\right]+\left\{\left[1-\frac{3}{5} \Phi_{p}^{2}(0)\right]^{2}+\frac{12}{5} \Phi_{p}^{2}(0) \exp \left(-\alpha_{x 0} l\right)\right\}^{1 / 2}}{\frac{6}{5}} .
$$

For small values of pump input the coefficient of the exponential may be considered to be small, and the square root in Eq. (53) may be expanded to obtain

$\Phi_{p}^{2}(l)=\frac{\Phi_{p}^{2}(0) \exp \left(-\alpha_{x 0} l\right)}{1-\frac{3}{5} \Phi_{p}^{2}(0)}-\frac{3}{5} \frac{\Phi_{p}^{4}(0) \exp \left(-2 \alpha_{x 0} l\right)}{\left[1-\frac{3}{5} \Phi_{p}^{2}(0)\right]^{3}}+\ldots$.

Keeping only terms to fourth power in $\Phi_{p}(0)$, this is

$\frac{\Phi_{p}^{2}(l)}{\Phi_{p}^{2}(0)}=\left(1+\frac{3}{5} \Phi_{p}^{2}(0)\left[1-\exp \left(-\alpha_{x 0} l\right)\right]\right) \exp \left(-\alpha_{x 0} l\right)$.

In terms of the intensity, Eq. (54) can be written

$\frac{I_{p}(l)}{I_{p}(0)}=\left(1+\frac{3}{5} s I_{p}(0)\left[1-\exp \left(-\alpha_{\lambda 0} l\right)\right]\right) \exp \left(-\alpha_{\lambda 0} l\right)$,

where $s$ is a saturation parameter incorporating the various coefficients given in Eq. (31). This result is compared with experimental data in the next section.

In a similar way one could also obtain an explicit analytic estimate of the dependence of the output signal intensity on the input pump intensity in the case of a ring laser oscillator. In most dye lasers the signal gain per pass is fairly small and one could repeat the integration of Eqs. (23) and (40) with $\Phi_{s}^{2}(z)$ assumed to be a nonzero constant. If the resulting expression for the pump intensity [replacing Eq. (55)] were substituted into Eqs. (24) and (41), one could integrate again to obtain a formula for the signal intensity. While the expected near-linear solutions of this type would be of some interest, they are not needed for the more qualitative interpretation of the experimental results reported here.

\section{EXPERIMENT}

A set of measurements has been carried out to see whether there are any discernible cross-saturation effects between the pump and signal fields in a dye laser amplifier. The laser system used in these measurements is similar to that described in a previously study. ${ }^{11}$ The dye laser is a vertical-jet $\mathrm{cw}$ unidirectional ring configuration based on a design developed at JILA, University of Colorado, ${ }^{13}$ and the optical pump source for the dye laser is the $514.5 \mathrm{~nm}$ line of a Spectra-Physics 2016 argon-ion laser. The pump and signal power levels are measured with a Coherent 210 power meter, and the overall setup is shown schematically in Fig. 2. The optical diode shown in the figure is a Faraday rotator followed by a wave plate, and this system serves to ensure unidirectional operation of the dye laser oscillator. The actual direction of signal propagation that one chooses is unimportant for this study as long as the detector is positioned properly and the signal gain per pass is small. The dye medium used in our experiments was a solution of rhodamine $6 \mathrm{G}$ in ethylene glycol at room temperature.

A typical set of experimental data is shown in Fig. 3. This plot shows the ratio of the transmitted pump power to the incident pump power as a function of the incident pump power for conditions of lasing and nonlasing at the signal wavelength of about $590 \mathrm{~nm}$. Nonlasing is obtained by blocking the internal beam path of the dye laser cavity. 


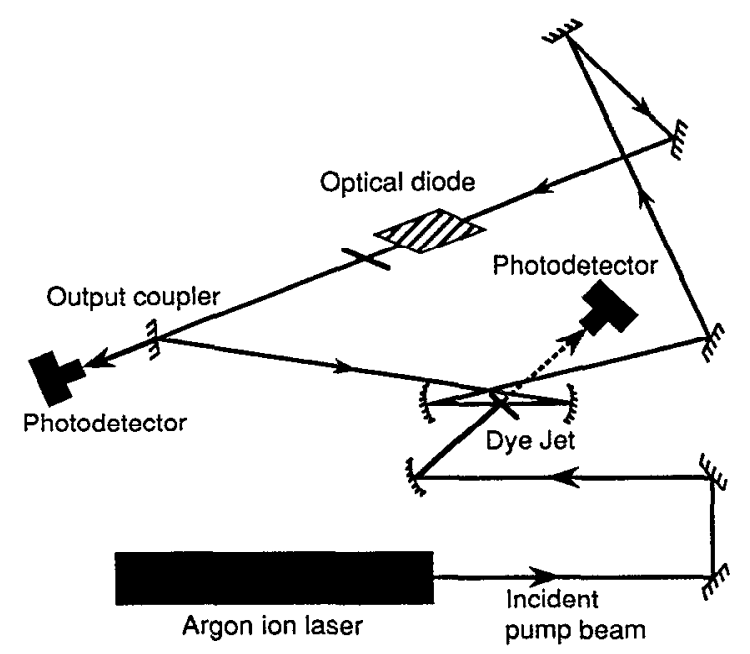

FIG. 2. Experimental unidirectional ring dye laser for cross-saturation studies.

This figure shows clearly our principal experimental result that the cross saturation by the laser field in a dye laser can have a significant effect on the absorption of the pump field. In this example, lasing with total cavity losses of about $6 \%$ limits the pump transmission to a value of about $11 \%$ rather than the nonlasing value of about $13 \%$. It is expected that this effect would be more dramatic with the higher signal circulating powers that would result from higher pump levels or from a reduction of signal losses.

It is also possible in principle to obtain more quantitative information about the interactions in the dye laser medium using the model that has been developed in the preceding section. It may be noted first of all that in the absence of lasing the pump transmission ratio increascs approximately linearly with the incident pump power as suggested by Eq. (56), except for a slight flattening at high

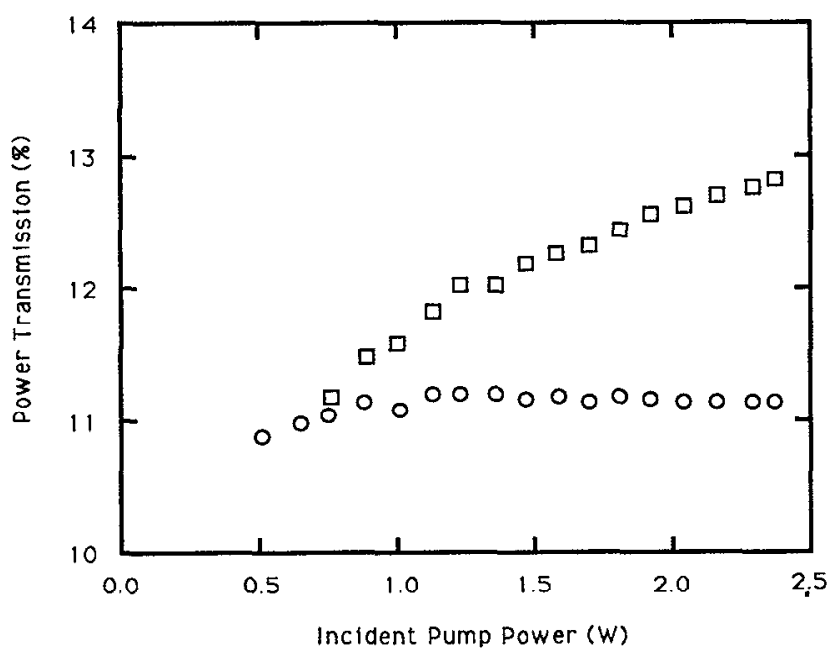

FIG. 3. Ratio of transmitted to incident pump power in the dye laser as a function of incident pump power for conditions of lasing $(O)$ and nonlasing $(\square)$ operation.

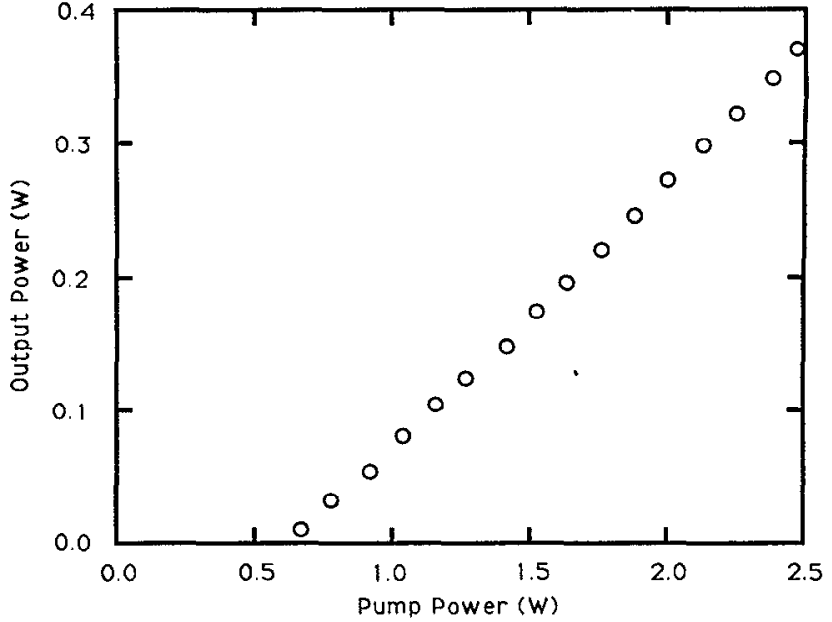

FIG. 4. Lasing output power as a function of pump input power.

pump levels. By extrapolating to zero incident pump intensity, one finds that the unsaturated pump transmission factor is about $\exp \left(-\alpha_{x 0} l\right)=0.10$. The thickness of the dye jet is roughly $100 \mu \mathrm{m}$, and hence the unsaturated absorption coefficient is about $\alpha_{x 0}=23000 \mathrm{~m}^{-1}$.

One can also use Eq. (56) to obtain information about the saturation parameter of the dye laser. The spot size of the pump beam at the dye jet can be inferred from the fact that it is focused on the jet with a mirror having a $7.5 \mathrm{~cm}$ radius of curvature. From the spot size and the pump power, the pump intensity can be estimated. Then the slope of the nonlasing curve in Fig. 3 can be used with Eq. (56) to obtain the saturation parameter $s$. If Eq. (56) is interpreted as a power equation rather than an intensity equation, one readily finds that the saturation parameter corresponding to the data shown in Fig. 3 is about $s=0.3 \mathrm{~W}^{-1}$.

The lasing threshold for the data in Fig. 3 occurs at about $0.65 \mathrm{~W}$ input power. After lasing commences the ratio of output pump power to incident pump power is essentially independent of the pumping level. This occurs because the lasing process more rapidly replenishes the lower laser state for further absorption of the pump beam, and it is consistent with the theoretical prediction summarized in Eq. (46). According to Eq. (46) the ratio of the transmitted pump intensity to the incident pump intensity is proportional to a power (near one) of the ratio of the transmitted signal intensity to the incident signal intensity. In a laser oscillator the ratio of transmitted to incident signal intensity is fixed by the cavity losses and, in particular, is independent of the level of the incident pump. Therefore, Eq. (46) predicts that the ratio of transmitted to incident pump should also be a constant, and from the data in Fig. 3 this constant is about $11 \%$.

We have also measured the lasing output as a function of the pump input power, and the resulting experimental data are shown in Fig. 4. The laser output power rises approximately linearly with the input pump power, and this behavior is consistent with the discussion of the previous section. The slope efficiency in this case is about $20 \%$. 


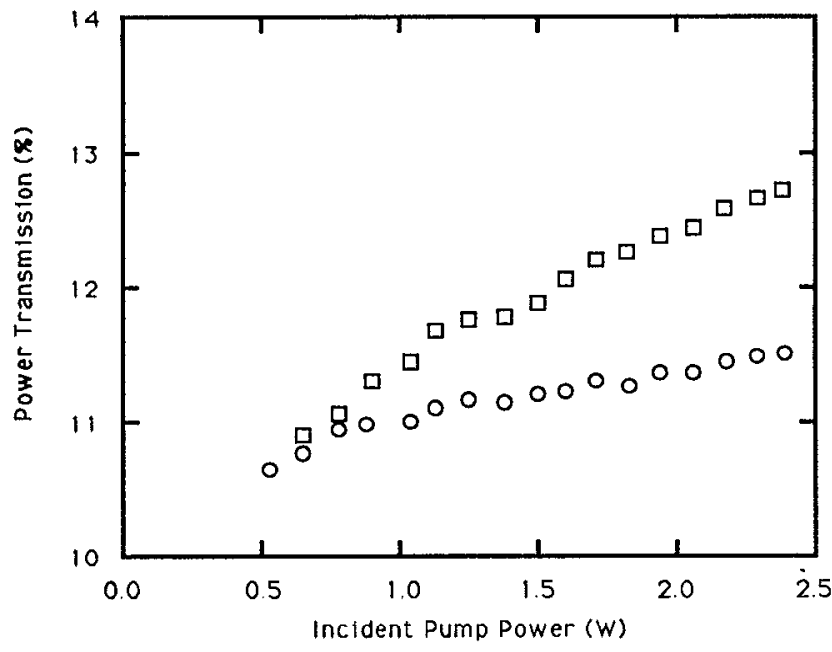

FIG. 5. Ratio of transmitted to incident pump power in the dye laser as a function of incident pump power for conditions of lasing $(O)$ and nonlasing ( $\square$ ) operation. In this example the pump beam area is larger than the signal beam area.

There are several geometrical factors that can weaken the cross-saturation effects being described here. First of all, it is possible that the pump and signal fields are not quite the same size at the dye jet. They have different wavelengths and in some cases different focusing mirrors. Beam overlap is also limited by the differing pump and signal astigmatisms and the fact that the pump and signal beams are not quite collinear. Finally, the fact that the beams are Gaussians rather than uniform plane waves as assumed in the analysis can reduce the accuracy of the saturation calculations. ${ }^{14}$ As an example, another set of pump transmission data is shown in Fig. 5, and the main change from Fig. 3 is that the pump in Fig. 5 has a softer focus, i.e., the pump beam at the dye jet is significantly larger than the signal beam. It is clear that in this case the saturation by the laser signal is less effective in limiting the pump transmission. Orientational relaxation is not included in our model and might also have an effect on the experimental results. ${ }^{11}$

\section{CONCLUSION}

In most treatments of $\mathrm{cw}$ laser oscillation it is assumed that the pump rate is a constant that depends only on the input source of pump power. However, there is always some more or less direct coupling of the energy states associated with the pump transition to the states associated with the lasing transition. Therefore, there is at least the possibility that a change in the lasing intensity might have some measurable effect on the transmitted pump intensity. The purpose of this study has been to address this question for the case of optically pumped dye lasers. It has been shown theoretically that for a typical dye laser the presence of a strong saturating signal tends to hold down the level of the transmitted pump intensity which otherwise would rise steadily as the input pump intensity is increased. This effect has also been shown experimentally for a one-directional ring dye laser oscillator. Varying the signal intensity in this laser leads to a modulation by several percent of the transmitted pump intensity. Measurements of this type can provide fundamental information about the parameters of the laser transition.

One can also imagine applications for the crosssaturation effect when one would otherwise lack a suitable nondeflecting modulator for an optical field. If that field could be used as the pump for a laser transition which possessed an appropriate level of cross saturation, then a wide variety of signal modulation techniques could be used indirectly to obtain the desired modulation of the original pump laser field. The speed of this modulation effect could also be far beyond conventional modulation rates if, for example, the secondary laser is mode locked to produce picosecond pulsations. In that case the transit of a picosecond signal pulse through the dye medium should be accompanied by a correspondingly fast drop in the transmitted pump intensity.

\section{ACKNOWLEDGMENTS}

This work was supported in part by the National Science Foundation under Grant No. ECS-9014481. The authors are also pleased to acknowledge valuable discussions with R. J. Ballagh and D. M. Warrington.

\section{APPENDIX: UNIDIRECTIONAL MOLECULAR DISTRIBUTION}

Most practical dye lasers have isotropic orientational distributions of the active molecular dipoles. However, it is also of interest to consider how the laser behavior might differ if all of the laser dipoles were oriented parallel to the pump and signal field polarizations. This limit is much easier to analyze and might also be realizable in some laser systems. With a unidirectional distribution the function $n(\theta, \phi)$ can be written

$$
n(0, \phi)=N \delta\left(\theta-\theta_{0}, \phi-\phi_{0}\right),
$$

where $\theta_{0}$ and $\phi_{0}$ represent the preferred orientation and the $\delta$ function is normalized by

$$
\int_{0}^{2 \pi} \int_{0}^{2 \pi} \delta\left(\theta-\theta_{0}, \phi-\phi_{0}\right) \sin \theta d \theta d \phi=1 .
$$

With Eq. (A1), the pump absorption and signal gain coefficients given in Eqs. (35) and (36) reduce to

$$
\begin{aligned}
& \alpha_{x}(z)=N \sigma_{p} D_{p}\left(\theta_{0}, \phi_{0}, z\right) \cos ^{2} \theta_{0}, \\
& g_{x}(z)=N \sigma_{s} D_{s}\left(\theta_{0}, \phi_{0}, z\right) \cos ^{2} \theta_{0} .
\end{aligned}
$$

When $\theta_{0}$ and $\phi_{0}$ are both zero, the molecular dipoles are all parallel to the pump and signal fields. Then with the population differences given in Eqs. (27) and (28), the absorption and gain coefficients in Eqs. (A3) and (A4) may be written explicitly as

$$
\alpha_{x}(z)=N \sigma_{p} \frac{1+\Phi_{s}^{2}(z)}{1+\left[\Phi_{p}^{2}(z)+\Phi_{s}^{2}(z)\right]},
$$




$$
g_{x}(z)=N \sigma_{s} \frac{\Phi_{p}^{2}(z)}{1+\left[\Phi_{p}^{2}(z)+\Phi_{s}^{2}(z)\right]} .
$$

As with the isotropic molecular distribution, these gain and loss expressions may be combined with Eqs. (23) and (24) to obtain a complete set of differential equations governing the pump and signal fields in the dye laser medium.

It follows from Eqs. (40) and (41) that the saturated absorption and gain coefficients in a laser amplifier with a unidirectional molecular distribution are related to each other by the formula

$$
\frac{\alpha_{x}(z)}{N \sigma_{p}}=1-\frac{g_{x}(z)}{N \sigma_{s}},
$$

and this result is similar to Eq. (42). As in the text, Eq. (A7) may be combined with Eqs. (23) and (24) to obtain the first integral

$$
\frac{\Phi_{p}^{2}(z)}{\Phi_{p}^{2}(0)}=\left(\frac{\Phi_{s}^{2}(z)}{\Phi_{s}^{2}(0)}\right)^{\sigma_{p} / \sigma_{s}} \exp \left(-\alpha_{x 0} z\right)
$$

where $\alpha_{x 0}=N \sigma_{p}$ is the unsaturated absorption coefficient from Eq. (A5). If the absorption and emission cross sections are considered to be equal, Eq. (A8) may be combined with Eqs. (24) and (A6) to obtain

$$
\frac{d \Phi_{s}^{2}(z)}{d z}=\alpha_{x 0} \frac{\left[\Phi_{p}^{2}(0) / \Phi_{s}^{2}(0)\right] \exp \left(-\alpha_{x 0} z\right)}{1+\left\{\left[\Phi_{p}^{2}(0) / \Phi_{s}^{2}(0)\right] \exp \left(-\alpha_{x 0} z\right)+1\right\} \Phi_{s}^{2}(z)} \Phi_{s}^{4}(z) .
$$

In terms of the new normalized length variable

$$
\zeta=\left[\Phi_{p}^{2}(0) / \Phi_{s}^{2}(0)\right] \exp \left(-\alpha_{x 0^{2}} z\right)+1,
$$

Eq. (A9) is

$$
\frac{d \Phi_{s}^{2}(\zeta)}{d \zeta}=-\frac{\Phi_{s}^{4}(\zeta)}{1+\zeta \Phi_{s}^{2}(\zeta)}
$$

It is now helpful to introduce the new field variable $F=\zeta \Phi_{s}^{2}(\zeta)$. With this substitution, Eq. (A10) separates into the form

$$
[(1+F) / F] d F=(1 / \zeta) d \zeta .
$$

This equation may be integrated to obtain an implicit analytical relationship between the $z$-dependent signal field and the input pump and signal fields in a dye laser amplifier. Using Eq. (A8), the corresponding $z$-dependent pump field can also be expressed analytically. This completeness of solution was not possible in the case of an isotropic orientational distribution discussed in the text.

${ }^{1}$ P. P. Sorokin, J. R. Lankard, E. C. Hammond, and V. L. Moruzzi, IBM J. Res. Dev. 11, 130 (1967).

${ }^{2}$ A. A. Kovalev and V. A. Pilipovich, Bull. Acad. Sci. USSR Phys. Ser. 34, 537 (1970).

${ }^{3}$ A. N. Sevchenko, A. A. Kovalev, and V. A. Pilipovich, Sov. Phys. Dokl. 14, 811 (1970).

${ }^{4}$ I. Nagata and T. Nakaya, J. Phys. D. 6, 1870 (1973).

${ }^{5}$ L. G. Pikulik and O. I. Yaroshenko, J. Appl. Spectrosc. 27, 861 (1977).

${ }^{6}$ L. G. Pikulik and O. I. Yaroshenko, J. Appl. Spectrosc. 27, 986 (1977).

${ }^{7}$ D. M. Phillion, D. J. Kuizenga, and A. E. Siegman, J. Chem. Phys. 61, 3828 (1974).

${ }^{8}$ K. C. Reyzer and L. W. Casperson, J. Appl. Phys. 52, 6075 (1980).

${ }^{9}$ K. C. Reyzer and L. W. Casperson, J. Appl. Phys. 52, 6083 (1980).

${ }^{10}$ L. I. Burov and I. I. Gancherenok, Opt. Spectrosc. (USSR) 61, 558 (1986).

${ }^{11}$ L. W. Casperson, W. J. Sandle, A. C. Wilson, D. M. Warrington, and R. J. Ballagh, J. Appl. Phys. 69, 8005 (1991).

${ }^{12}$ Y. B. Band and D. F. Heller, Phys. Rev. A 38, 1885 (1988).

${ }^{13}$ J. L. Hall (private communication).

${ }^{14}$ L. W. Casperson, Appl. Opt. 19, 422 (1980). 